\title{
ARTICLE OPEN Topological defects at octahedral tilting plethora in bi-layered perovskites
}

\author{
Fei-Ting Huang ${ }^{1}$, Bin Gao ${ }^{1}$, Jae-Wook Kim ${ }^{1}$, Xuan Luo $^{2}$, Yazhong Wang ${ }^{1}$, Ming-Wen Chu ${ }^{3}$, Chung-Kai Chang ${ }^{4}$, Hwo-Shuenn Sheu ${ }^{4}$ and \\ Sang-Wook Cheong ${ }^{1,2}$
}

Oxygen octahedral distortions, including tilts/rotations, deformations and off-centring in (layered) perovskites, have the key role in their numerous functional properties. Near the polar-centrosymmetric phase boundary in bi-layered perovskite $\mathrm{Ca}_{3}-\mathrm{Sr}_{x} \mathrm{Ti}_{2} \mathrm{O}_{7}$ with $x \approx 1$, we found the presence of abundant topological eight-state vortex-antivortex pairs, associated with four oxygen octahedral tilts at domains and another four different oxygen octahedral tilts at domain walls. Our discovery opens a new revenue to unveil realspace topological defects associated with the possible vector choices in one specific lattice mode.

npj Quantum Materials (2016) 1, 16017; doi:10.1038/npjquantmats.2016.17; published online 7 October 2016

\section{INTRODUCTION}

Copious functional phenomena, including high $T_{\mathrm{c}}$ superconductivity, ${ }^{1}$ ferroelectricity, ${ }^{2,3}$ novel magnetism ${ }^{4-6}$ and giant photovoltaic effects, ${ }^{7,8}$ have been observed in perovskite $\left(\mathrm{ABO}_{3}\right)$-related compounds, where those physical properties can be closely associated with oxygen octahedral distortions, including tilts/rotations, deformations and off-centring. For example, the high carrier mobility in transparent conducting cubic $\mathrm{BaSnO}_{3}$ (ref. 9) or photovoltaic halide perovskites ${ }^{8,10}$ is closely related with (nearly) $180^{\circ}$ bonding between large B-site cations and an oxygen (or a halide ion), resulting from little octahedral distortions. Even when A-site ions are rather small, the perovskite-related structure can be still stabilised through oxygen octahedral tilts/rotations. ${ }^{11}$ It turns out that superconductivity in $(\mathrm{La}, \mathrm{Ba})_{2} \mathrm{CuO}_{4}$ is significantly influenced by oxygen octahedral tilts, ${ }^{12,13}$ and canted magnetic moments appear in antiferromagnetic perovskites with tilted/ rotated oxygen octahedra through Dzyaloshinskii-Moriya interaction. ${ }^{2,14}$ High dielectric response in the vicinity of the morphotropic phase boundary is a consequence of the continuously rotating polarisation with various octahedral tilts. ${ }^{15,16}$ Crystal field split can be considerably influenced by compression or elongation of oxygen octahedra, which is the origin of the Jahn-Teller effects for $\mathrm{B}=\mathrm{Cu}^{2+}$ or $\mathrm{Mn}^{3+}$ (refs $1,17,18$ ).

Remarkably, the simultaneous presence of oxygen octahedral tilt and rotation can result in ferroelectric polarisation in perovskites with even number of layers, which is called hybrid improper ferroelectricity. ${ }^{19-21}$ It has experimentally verified that bilayered perovskite $\mathrm{Ca}_{3-x} \mathrm{Sr}_{x} \mathrm{Ti}_{2} \mathrm{O}_{7}$ (CSTO) is a hybrid improper ferroelectric with switchable polarisation of $8 \mu \mathrm{C} \mathrm{cm}^{-2}$ in bulk crystals at room temperature. ${ }^{20}$ Ferroelectricity in CSTO described by a hybridisation of two structural modes (octahedral tilt and rotation modes) turns out to be associated with an intriguing domain topology consisting of $Z_{4} \times Z_{2}$ domains and $Z_{3}$ vortices with eight domains (four directional domains and two antiphase domains), abundant charged domain walls and unique zipper-like switching kinetics. ${ }^{22}$ In $Z_{4} \times Z_{2}$ domains, $Z_{4}$ denotes the cyclic group of order 4 for directional variants and $Z_{2}$ is for translational variants. In this article, utilising in situ heating transmission electron microscopy (TEM) studies, synchrotron powder X-ray diffraction experiments and dielectric measurements, we report the discovery of a new intermediate tetragonal state in CSTO, which demonstrates a displacive nature of hybrid improper ferroelectricity mechanism different from those predicted from the group-subgroup relation. ${ }^{21,23}$ Furthermore, we find the presence of topological eight-state vortex-antivortex defects associated with two-dimensional eight degrees of freedom for oxygen octahedral tilts in the intermediate tetragonal state.

\section{RESULTS}

Figure 1 depicts the possible octahedral tilts/rotations in bi-layered perovskite CSTO, resulting in different structural states and also domains with different directional order parameters. For each space group, the distortion axes and the corresponding Glazer notations ${ }^{24}$ are given with respect to the un-distorted tetragonal $14 / \mathrm{mmm}$ (T) structure (Figure 1b). The term 'rotation' denotes a rotation of the basal oxygen plane around the [001] axis in a clockwise (+) or counterclockwise (-) manner (Figure 1c for +). Out-of-phase and in-phase rotation in adjacent layers within one bi-layered perovskite block lead to orthorhombic $\mathrm{O}^{*}$ and $\mathrm{O}^{\prime}$ states, respectively. Note that the presence of an intermediate $\mathrm{O}^{*}$ state (Figure 1c) competing with the ground-state polar $\mathrm{O}$ state, responsible for uniaxial negative thermal expansion, has been confirmed in hybrid improper ferroelectricity magnet $\mathrm{Ca}_{3} \mathrm{Mn}_{2} \mathrm{O}_{7}$ (refs 25,26$)$. The term 'tilt' is associated with a tilt around an inplane axis, and it would move the basal oxygens out of the basal plane and the apical oxygen away from the $c$ axis. In-plane tilting axes can be along $\langle 110\rangle_{\mathrm{T}}$ and $\langle 100\rangle_{\mathrm{T}}$ directions, leading to orthorhombic $\mathrm{O}^{\prime}$ (Figure 1e) and tetragonal $\mathrm{T}^{\prime}$ states (Figure 1f), respectively. Following the Glazer notation, the $T$ state has the $a^{0} a^{0} c^{0}$ pattern and the $\mathrm{T}^{\prime}, \mathrm{O}^{\prime}, \mathrm{O}^{*}$ and $\mathrm{O}^{\prime \prime}$ states are described as $a^{-} a^{0} c^{0}, a^{-} a^{-} c^{0}, a^{0} a^{0} c^{-}$and $a^{0} a^{0} c^{+}$, respectively. Two end members, $\mathrm{Sr}_{3} \mathrm{Ti}_{2} \mathrm{O}_{7}$ and $\mathrm{Ca}_{3} \mathrm{Ti}_{2} \mathrm{O}_{7}$, correspond to two extremes: the un-distorted $\mathrm{T}$ state of $\mathrm{Sr}_{3} \mathrm{Ti}_{2} \mathrm{O}_{7}$ and the $\mathrm{O}$ state with both tilt

\footnotetext{
${ }^{1}$ Rutgers Center for Emergent Materials and Department of Physics and Astronomy, Rutgers University, Piscataway, New Jersey, USA; ${ }^{2}$ Laboratory for Pohang Emergent Materials and Max Plank POSTECH Center for Complex Phase Materials, Pohang University of Science and Technology, Pohang, Korea; ${ }^{3}$ Center for Condensed Matter Sciences, National Taiwan University, Taipei, Taiwan and ${ }^{4}$ National Synchrotron Radiation Research Center, Hsinchu, Taiwan.

Correspondence: S-W Cheong (sangc@physics.rutgers.edu)

Received 10 June 2016; revised 27 July 2016; accepted 16 August 2016
} 


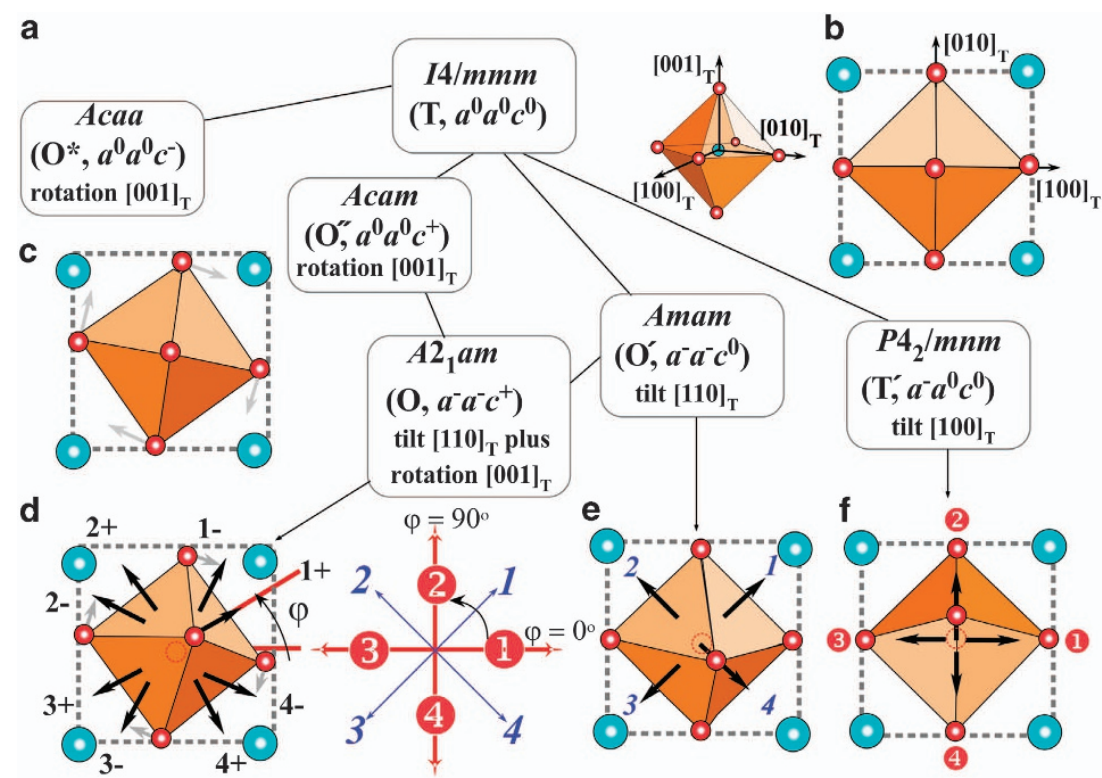

Figure 1. (a) Schematic diagram of possible space groups and the corresponding Glazer notations for bi-layered perovskite $A_{3} B_{2} \mathrm{O}_{7}$. Lines link the group-subgroup relations. The lattice directions are given with respect to the T state $(14 / \mathrm{mmm})$. (b-f) In-plane projected views of various $\mathrm{BO}_{6}$ octahedral tilts/rotations. The dashed lines outline the primitive unit cells, and the red and cyan spheres represent the $\mathrm{O}$ and $\mathrm{B}$-site ions, respectively. Black and gray arrows indicate directions of apical and basal plane oxygen displacements, respectively. (b) Un-distorted $\mathrm{BO}_{6}$ octahedron in the T state. (c) A clockwise $(+)$ rotated $\mathrm{BO}_{6}$ in the $\mathrm{O}^{*}$ and $\mathrm{O}^{\prime}$ states. The rotation can be also counterclockwise $(-)$. In two adjacent layers within one bi-layer, an out-of-phase rotation $(+-$ or -+$)$ along the $c$ axis occurs in the $\mathrm{O}^{*}$ state while in-phase $(++$ or --$)$ rotation in the $\mathrm{O}^{\prime \prime}$ state. (d) Eight possible $1 \pm, 2 \pm, 3 \pm$ and $4 \pm$ apical oxygen displacements in the $O$ state. The azimuthal angle, $\varphi$, of an apical oxygen distortion is denoted. (e) Given tilting vectors in the $\mathrm{O}^{\prime}$ state. (f) Four possible apical oxygen displacements (red-circled labels) along the $<100>_{\mathrm{T}}$ directions in the $\mathrm{T}^{\prime}$ state.

and rotation $\left(a^{-} a^{-} c^{+}\right)$of $\mathrm{Ca}_{3} \mathrm{Ti}_{2} \mathrm{O}_{7}$ (Figure $1 \mathrm{~d}$ ). It is clear why orthorhombic $\mathrm{O}^{\prime}\left(a^{-} a^{-} c^{0}\right)$ and $\mathrm{O}^{\prime \prime}\left(a^{0} a^{0} c^{+}\right)$states are the two plausible intermediate structures toward the polar $\mathrm{O}$ structure $\left(a^{-} a^{-} c^{+}\right)$by symmetry. ${ }^{21,23}$ With the underlying square lattice, various symmetry-equivalent domains (phases) may exist in each of those states. It is convenient to define the azimuthal angle of an apical oxygen distortion, $\varphi$, as shown in Figure $1 \mathrm{~d}$. This $\varphi$ links all possible directions of apical oxygen motions among those phases; for example, symmetry-equivalent domains of the $\mathrm{T}^{\prime}$ phase correspond to $\varphi=0^{\circ}, 90^{\circ}, 180^{\circ}$ and $270^{\circ}$ (red-circled $\mathbf{0}, \mathbf{2}, \mathbf{3}$ and 4 in Figure 1f, respectively), whereas those in the $\mathrm{O}^{\prime}$ phase to $\varphi=45^{\circ}, 135^{\circ}, 225^{\circ}$ and $315^{\circ}$ (blue, 1, 2, 3 and 4 in Figure 1e, respectively). The domains of the polar $O$ state has $\varphi=45^{\circ} \pm a$, $135^{\circ} \pm a, 225^{\circ} \pm a$ and $315^{\circ} \pm a$, where $a$ depends on the sign and magnitude of octahedral rotation $\left(a^{0} a^{0} c^{+} ; 1 \pm, 2 \pm, 3 \pm\right.$ and $4 \pm$ in Figure $1 \mathrm{~d}$, respectively).

We have performed synchrotron powder X-ray diffraction experiments using the collimated synchrotron-radiation beam with the wavelength of $0.688 \AA$ at the National Synchrotron Radiation Research Center, Taiwan. Homogeneous and phase-pure polycrystalline CSTO $(0 \leqslant x \leqslant 3)$ specimens were prepared by a solid-state reaction method (Materials and methods). The general structure analysis system program using the Rietveld method with a pseudo-Voigt profile function was exploited to analyse the powder diffraction data. The evolution of octahedral rotation $\left(\theta_{R}\right)$ and tilting $\left(\theta_{\mathrm{T}}\right)$ angles of $\mathrm{TiO}_{6}$, and lattice parameters $a, b$ and $c$ as a function of Sr content, $x$, is summarised in Figure $2 \mathrm{a}$ and Supplementary Figure S1a. The phase diagram, constructed from these structural parameters, consists of ferroelectric $O$ (purple, $0 \leqslant x \leqslant 0.9$ ) and paraelectric T states (pink, $x \geqslant 1.5$ ). The asymmetric decays of $\theta_{\mathrm{R}}$ and $\theta_{\mathrm{T}}$ at $x=0.915-1$ defines a sharp and narrow region with only non-zero $\theta_{\mathrm{T}}$ (yellow, Figure $2 \mathrm{a}$ ). The highresolution X-ray diffraction data clearly display peak splitting only when $x \leqslant 0.9$, implying a tetragonal symmetry in this narrow region (Supplementary Figure S1b). A $\sqrt{2} \times \sqrt{2}$ supercell in electron diffraction patterns from TEM (Figure 2c) is found for $x=0.915-1$, confirming the stabilisation of a new tetragonal intermediate state distinct from the parent $T$ state. The Rietveld refinement of synchrotron data provides further confirmation of the existence of the $\mathrm{T}^{\prime}$ state with the $a^{-} a^{0} c^{0}$ pattern (Supplementary Figure S1c). The details of X-ray refinement fits are given in Supplementary Section 1 and Supplementary Table S1. The dielectric constant also shows a marked change in magnitude upon entering the $\mathrm{T}^{\prime}$ state and displays almost two times larger epsilon $(\varepsilon)$ value at the phase boundary of $x=0.9$, compared with that of low $x$ values (Figure 2b). An increase in $\varepsilon$ at $350 \mathrm{~K}$ can be understood as increasing structural fluctuations when approaching from the ferroelectric $\mathrm{O}$ to paraelectric $\mathrm{T}^{\prime}$ states. Indeed, in situ TEM heating experiments of $\mathrm{Ca}_{2.1} \mathrm{Sr}_{0.9} \mathrm{Ti}_{2} \mathrm{O}_{7}$ crystals exhibit a two-step transformation upon heating: $\mathrm{O} \rightarrow \mathrm{T}^{\prime} \rightarrow \mathrm{T}$. Figure $2 \mathrm{c}$ shows first that the intensity of superlattice $S_{1}$-type spots $1 / 2(130)_{T}$ of the $O$ state (cyan triangles) weakens as temperature ( $T$, defined italic $T$ as temperature) is raised from 300 to $450 \mathrm{~K}$. Two additional sets of superlattice $S_{2}$-type $1 / 2(200)_{T}$ and $S_{3}$-type $1 / 2(\overline{1} 30)$ spots (yellow and green triangles), corresponding to the $\mathrm{T}^{\prime}$ state, appear when temperature is further raised to $473 \mathrm{~K}$. Finally, all superlattice spots vanish above $713 \mathrm{~K}$. Thus, we have demonstrated the stabilisation of the intermediate $\mathrm{T}^{\prime}$ state by varying chemical composition as well as temperature. Starting from the $\mathrm{O}$ state at low $x$, Figure $2 \mathrm{a}$ shows a faster relaxation of the octahedral rotation $\left(\theta_{R}\right)$ than that of tilting $\left(\theta_{T}\right)$ with increasing $\mathrm{Sr}$ doping, which is also coupled with an increasing trend of orthorhombicity. ${ }^{20}$ The sudden suppression of octahedral rotations occurs when the azimuthal angle $\varphi$ suddenly switches from $\sim 45^{\circ}$ at $x=0.9$ to $0^{\circ}$ in the $T^{\prime}$ state (Figure 1a), indicating a likely discontinuous change of the tilting order parameter and a first-order phase transition. Note that we do not observe any evidence for an intermediate 
a

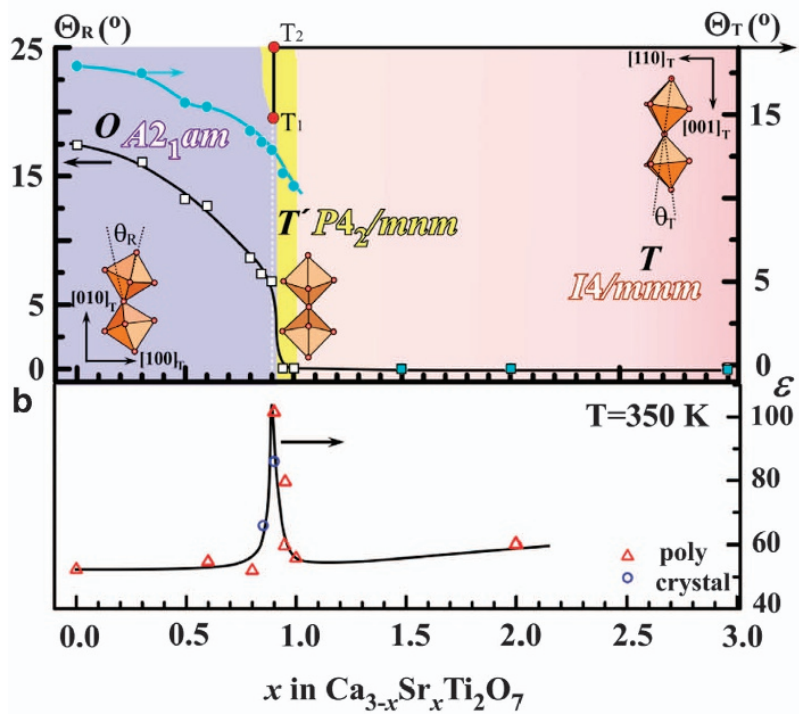

C

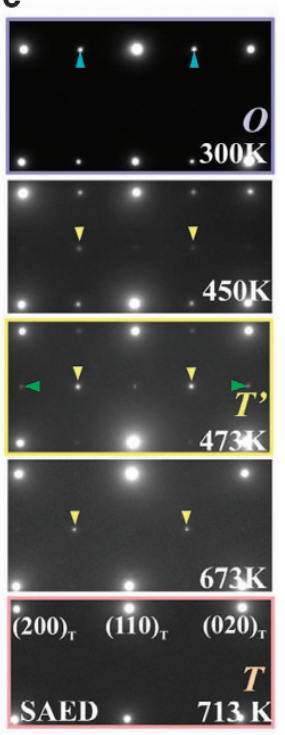

Figure 2. (a) Structural phase diagram of $\mathrm{Ca}_{3}{ }_{-} \mathrm{Sr}_{x} \mathrm{Ti}_{2} \mathrm{O}_{7}$ with the angles of octahedral rotation $\left(\theta_{\mathrm{R}}\right.$, empty squares) and tilt $\left(\theta_{\mathrm{T}}\right.$, cyan spheres) as a function of $\mathrm{Sr}$ doping, $x$. The black and cyan curves are guides for the eyes. Two structural phase transition temperatures $\left(T_{1}=473 \mathrm{~K}\right.$ and $T_{2}=710 \mathrm{~K}$ ) at $x=0.9$, marked with red spheres, are obtained from in situ TEM heating experiments. (b) Dielectric constant versus Sr content ( $x$ ), measured at $350 \mathrm{~K}$ and $44 \mathrm{kHz}$. Red triangles and blue circles are from measurements on polycrystalline and single-crystalline (electric field applied along the in-plane direction) specimens, respectively. The dielectric constant peaks at the O-T' structural boundary of $x=0.9$. (c) Thermal sequence of selected area electron diffraction patterns on $x=0.9$, showing two phase transitions upon in situ heating: $\mathrm{O}$ ( $300 \mathrm{~K}) \rightarrow \mathrm{T}^{\prime}$ $(473 \mathrm{~K}) \rightarrow \mathrm{T}(713 \mathrm{~K})$ states. $S_{1}=1 / 2(130)_{T}$-type superlattice spots (cyan triangles) are allowed in the $\mathrm{O}$ state. When temperatures reaches $473 \mathrm{~K}$, additional $S_{2}=1 / 2(200)_{T}$-type and $S_{3}=1 / 2(-130)_{T}$-type superlattice spots appear (yellow and green triangles), indicating the appearance of a tetragonal $\mathrm{T}^{\prime}$ state. Above $713 \mathrm{~K}$, all superlattice spots disappear, consistent with the presence of the $\mathrm{T}$ state at very high temperatures.

$\mathrm{O}^{\prime}\left(a^{-} a^{-} c^{0}, \varphi=45^{\circ}\right)$ state, reported in the solid solution of $\mathrm{Ca}_{3} \mathrm{Ti}_{2} \mathrm{O}_{7}-(\mathrm{Ca}, \mathrm{Sr})_{1.15} \mathrm{~Tb}_{1.85} \mathrm{Fe}_{2} \mathrm{O}_{7}$ (ref. 27).

Figures $3 a$ and $b$ show a $2.7 \times 1.7 \mu \mathrm{m}$ mosaic of dark-field (DF) TEM images of the $\mathrm{T}^{\prime}$ state $x=0.95$ taken using $\mathrm{S}_{2}$ spot (red circle in Figure $3 \mathrm{~b}$ ) and its corresponding diffraction pattern along the $[001]_{T}$ direction. The curved dark-contrast lines reveal boundaries of four $\mathrm{T}^{\prime}$-phase domains merging at one core, which is a non- $\mathrm{T}^{\prime}$ state. With the underlying square lattice, 4 symmetry-equivalent domains, named $Z_{1} \times Z_{4}$ domains, may exist; four translational variants associated with the translation vectors $(1 / 2,1 / 2,0),(0,1 / 2,1 / 2)$ and $(1 / 2,0,1 / 2)$ where out-of-plane phase shifts are involved in the latter two. However, the domain topology can be renamed as $Z_{2} \times Z_{2}$ domains when the in-plane order parameters (octahedral tilts) of one bilayer is considered; two directional variants $\left([010]_{\mathrm{T}^{-}}\right.$ tilt producing $\mathbf{0} / \mathbf{3}$ and $[100]_{T}$-tilt producing $\left.\mathbf{2} / \mathbf{4}\right)$ and two translational variants associated with the in-pane translation vector $(1 / 2,1 / 2,0)$ between (1-3 and 2-4 (Figure 1f). Our results demonstrate that four domains, corresponding to red-circled $\mathbf{0}$, 2, 3 and 4 in Figure 1f, form an exclusive vortex-like pattern with $90^{\circ}$-rotating apical oxygen distortions (red arrows in Figure 3a). These four domains are represented by four values of apical oxygen azimuthal angles $\varphi=0^{\circ}, 90^{\circ}, 180^{\circ}$ and $270^{\circ}$; for example, domains $\left(1\right.$ and 3 correspond to $\varphi=0^{\circ}$ and $180^{\circ}$, respectively. Note that the domain network (Figure 3a) can be described by two proper colouring, i.e., two colours is sufficient to identify the domains without neighbouring domains sharing the same colour (white and green in Supplementary Figure S3a), and the vortex network can be constructed by cutting through two types of closed loops (blue and light blue in Supplementary Figure S3a) based on a graph-theoretical description. ${ }^{28}$ Intriguingly, those curved domain walls exhibit two distinct extinction rules in the superlattice DF-TEM images. Figure $3 c$ shows a DF-TEM image using a $S_{1}$ spot (light-blue circled in Figure $3 b$ ), revealing only a part of domain walls. A complementary domain-wall map is obtained using a $S_{3}$ spot for DF-TEM imaging (blue-dotted circled in Figure $3 b$ ). The inequivalent nature of these two DF-TEM images with different superlattice peaks with $a b$ plane components indicates an orthorhombic-like local structure at those walls, instead of, e.g., a high-symmetry $T$ state. Considering a pure tilting nature of the matrix $\mathrm{T}^{\prime}$ state and the distinct extinction rules, those domain walls are likely in the $\mathrm{O}^{\prime}$ state (Figure 1e). The directions of oxygen octahedral tilts and apical oxygen distortions are denoted as light blue and blue arrows in the Figures $3 c$ and $d$ insets, respectively - more details are discussed in Supplementary Section 2. Therefore, oxygen octahedra inside the brightcontrast domains tilt along either $[100]_{T}$ or $[010]_{T}$ directions whereas those in the dark-contrast domain walls tilt along diagonal $\langle 110\rangle_{\mathrm{T}}$ directions. The full assignment of vortices and antivortices in Figure $3 a$ is given in Supplementary Figures S3a and $b$, based on the structural information derived from Figures $3 c$ and $d$. This leads to a unique $Z_{4}$ vortex structure with four domains and four domain walls, where oxygen octahedra tilt and $\varphi$ changes by $45^{\circ}$ consecutively around the vortex core. The $\mathrm{Z}_{4}$ vortex consists of four phases of domains and also four other phases at domain walls allowed with $X_{3}^{-}$mode representation of octahedral tilts, whereby the tilting axes and amplitude are specified by the order parameter direction. The relevant order parameter directions are $(a, a)$ for the T'-state and $(a, 0)$ for the O'state, where the value $a$ corresponds to the magnitude of tilt around the $x$ and $y$ axes. ${ }^{29}$ Indeed, those domain walls show a finite width of $\sim 7-9$ T'-unit cells as estimated from a scanning TEM image (yellow-shaded area in Figure 3e), which is much thicker than that of atomically sharp domain walls found in proper ferroelectrics such as in $\mathrm{Pb}\left(\mathrm{Zr}_{0.2} \mathrm{Ti}_{0.8}\right) \mathrm{O}_{3}$ (ref. 30). The structural model superimposed in Figure 3 e corresponds to one projected unit cell (blue: $\mathrm{Ca} / \mathrm{Sr}$; green: Ti; grey: $\mathrm{Sr}$; and red: O). The A-site 

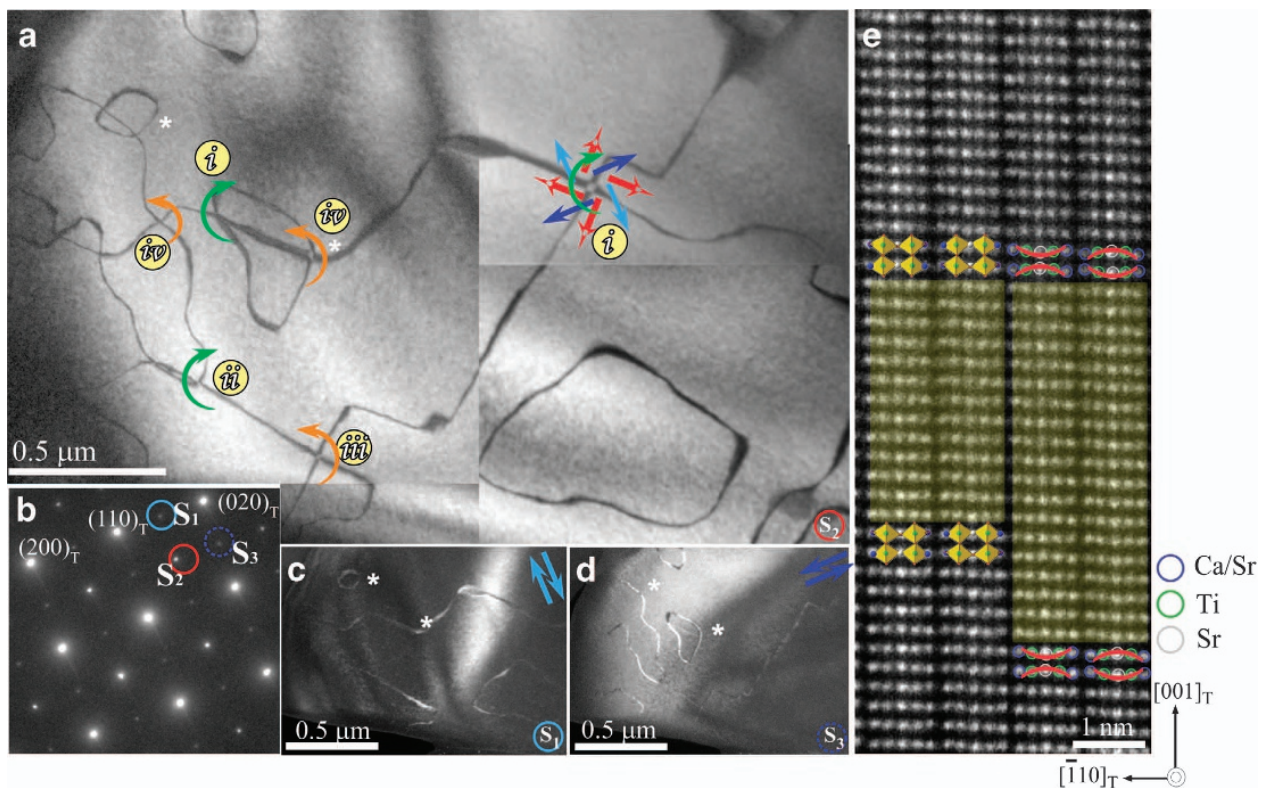

Figure 3. (a) A $2.7 \times 1.7 \mu \mathrm{m}$ mosaic of DF-TEM images, taken using a superlattice $\mathrm{S}_{2}$ spot (red circled) in a CSTO $x=0.95$ specimen along the $[001]_{T}$ direction. The coloured arrows represent octahedral tilting directions; red arrows for four phases of domains in the $T^{\prime}$ state and (light)blue arrows for four phases of domain walls in the $\mathrm{O}^{\prime}$ state. Yellow-circled $i$-iv are $\mathrm{Z}_{4}$ vortex-antivortex defects. Asterisks are location markers. (b) The corresponding selected area electron diffraction. (c) A superlattice DF-TEM image, taken using a superlattice $S_{1}$ spot (light blue-circled), exhibits only a part of domain walls in a. (d) A superlattice DF-TEM image, taken using a $\mathrm{S}_{3}$ spot (blue-dot circled), shows the rest of domain walls. Octahedral tilting directions in real space are shown in the up-right corner. (e) A high-angle annular dark-field image of one domain wall acquired along $[110]_{\mathrm{T}}$. The superimposed structural model corresponds to one projected unit cell. Ca/Sr, Ti and Sr cations are shown with blue, green and grey spheres, respectively. The unit cells can be determined with the brackets '(' or ')' geometry of cation displacements. The number of brackets between two highlighted unit cells is an odd number, evidencing a $\pi$ phase shift and the existence of an APB. A less distortion is evident in the yellow-shaded APB region.

cation ordering is also confirmed from the observation of a brighter contrast of perovskite layers than that of rock-salt layers (Figure 3e). The observation of eight-state vortex-antivortex defects composed of $\mathrm{T}^{\prime}$ and $\mathrm{O}^{\prime}$ state suggests an $\mathrm{X}_{3}^{-}$modedriven phase transition.

Figure 4a summarises two types of $Z_{4}$ vortices (types $i$ and ii) and two types of $Z_{4}$ antivortices (types iii and $i v$ ) observed in Figure 3a. Topological charge or winding number ' $n$ ' is assigned when vectors rotate clockwise by $2 \pi n$ along the clockwise direction around a core. Then, the topological charges of types $i$, ii, iii and iv topological defects are $+1,+1,-1$ and -1 , respectively, which indicates that types $i$ and $i i$ are vortices and types iii and iv are antivortices. We emphasise that vectors in a type $i$ topological defect rotate opposite to those in a type ii topological defect, but they have the same topological charge, so both of them are vortices. $\mathrm{A} \mathrm{Z}_{4}$ vortex is always surrounded by antivortices and vice versa; for example, the vortex ' $l$ ' in Figure $3 a$ is connected to the antivortex 'iii' and the antivortex ' $i v$ '. Figure $4 \mathrm{~b}$ illustrates the local structure around a type $i Z_{4}$ vortex (Figure $4 a$ ) with red-circled $\mathbf{0}$, 2, 3 and 4 domains, and domain walls of DW02 (blue-broken lines), DW04 (light-blue-broken), DW34 (blue) and DW23 (light blue). By averaging the structure of neighbouring domains, DWO2 and DWB4 share the same $[110]_{\mathrm{T}}$-tilting axis, but are different in the origin by a half of the unit cell. Similarly, DW23 and DWO4 have the same tilting axis, which is consistent with the extinction rules observed in Figures $3 c$ and $d$. On the other hand, a geometric frustration of oxygen octahedral distortions between two antiphase domains occurs likely in DWO3 (Figure 4c), leading to an undistorted domain wall in a T structure-like high-symmetric state. The absence of $Z_{3}$ vortices where three types of domain walls merge at one point reveals the nonexistence of $(1 / 2,1 / 2,0)$ type APB such as DW03, which, in turn, indicates a much higher energy associated with APBs.

\section{DISCUSSION}

The $\mathrm{T}^{\prime}$ state showing $\mathrm{Z}_{4}$ vortices occurs in a narrow compositional range of $\left(\mathrm{Ca}, \mathrm{Sr}_{3} \mathrm{Ti}_{2} \mathrm{O}_{7}\right.$. When octahedral rotation (thus, hybrid improper ferroelectricity) is suppressed by chemical doping/ionic ordering, $Z_{4}$ vortices emerge in the narrow compositional range, where domain and domain walls are intricately intertwined with the $\mathrm{T}^{\prime}$ and $\mathrm{O}^{\prime}$ states, arising from the active $\mathrm{X}_{3}^{-}$mode. A full isotropy subgroup analysis ${ }^{29}$ further implies a missing intermediate orthorhombic Pnnm state, representing order parameter direction $(a, b)$, which is a subgroup of both of the $\mathrm{T}^{\prime}$ and $\mathrm{O}^{\prime}$ states, and is expected by symmetry to link domain and domain walls. A similar rule has been also applied to topological $Z_{6}$ vortices in hexagonal system, $h-\operatorname{In}\left(\mathrm{Mn}_{0.9} \mathrm{Ga}_{0.1}\right) \mathrm{O}_{3}$, where the $\mathrm{K}_{3}$ mode is characterised by trimerising tilts of the $\mathrm{MnO}_{5}$ trigonal bipyramids. Three low-symmetry possibilities derived from the parent $P \sigma_{3} / m m c$ structure are allowed by the $K_{3}$ mode, corresponding to 12 phases of domains and domain walls and an intermediate state link them. ${ }^{31}$ Real-space topological defects can be well classified in terms of one specific lattice mode, which leads to a valid physical insight into the phase transition and serves as a platform to uncover additional hidden symmetry and new topological defects. In addition, our new discovery of ' $Z_{2} \times Z_{2}$ domains with $Z_{4}$ vortices' unveils that there can be a lot more than just the topological defects $\left(Z_{2} \times Z_{3}\right.$ domains with $Z_{6}$ vortices) in hexagonal rare-earth manganites, which has been extensively investigated since the initial discovery in 2010 (ref. 32). $Z_{6}$ vortices observed in improper ferroelectric rare-earth manganites ${ }^{31,32}$ and skyrmions in low-symmetry magnets ${ }^{33}$ have provided a new paradigm in the quest for mesoscopic self-organised structures with non-trivial topology, which can have novel functionalities. Emphasise that the octahedral distortions in the $T^{\prime}$ state is also analogous to that observed in the so-called low-temperature 
a

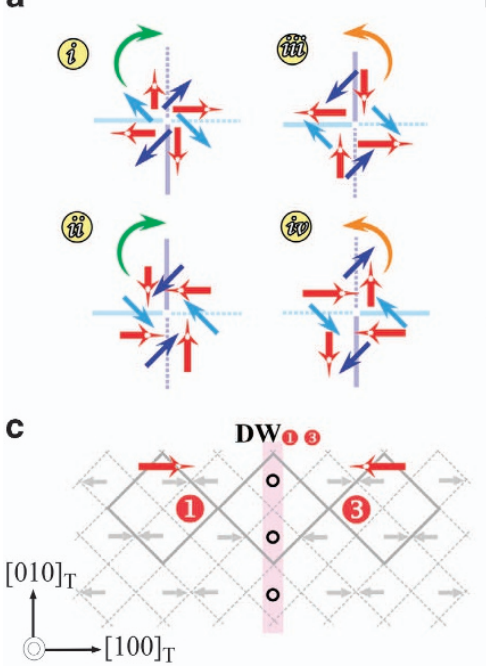

b

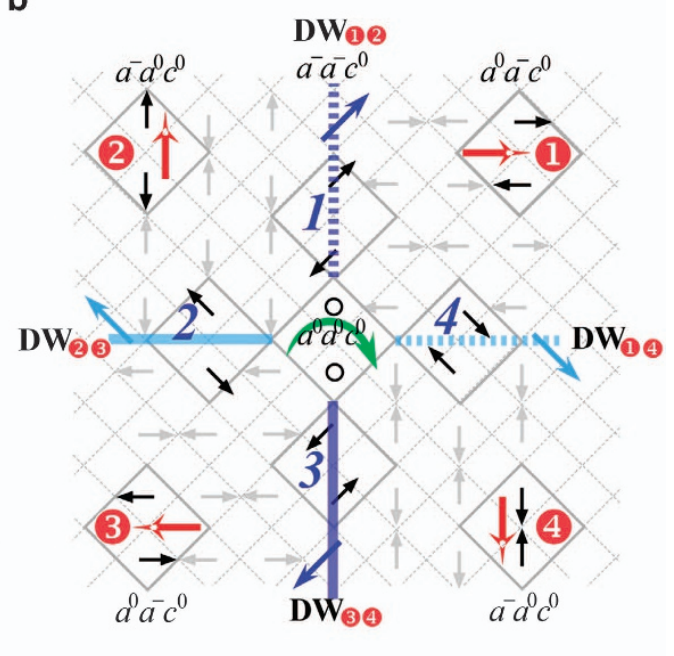

Figure 4. (a) Schematics illustrate the oxygen octahedral distortion configurations at domains and domain walls of $Z_{4}$ vortex-antivortex defects shown in Figure 3a. Red arrows for four domains show apical oxygen tilts along the $\langle 100\rangle_{\mathrm{T}}$ directions. Blue and light-blue bold lines represent two types of domain walls originated from two different tilting axes, and solid and broken lines are related by a translational symmetry. Yellow-circled types $i$ and $i i$ are $Z_{4}$ vortices and types iii and $i v$ are $Z_{4}$ antivortices. (b) Local oxygen distortions of a type $i Z_{4}$ vortex with four domains (red-circled (-4) and four domain walls (blue 1-4) in the $a b$ plane. The local apical oxygen distortions in each domain and domain wall are shown by small gray and black arrows. The gray solid squares outline the $\sqrt{2} \times \sqrt{2}$ supercell with the $\mathrm{T}^{\prime}\left(P 4_{2} / \mathrm{mnm}^{\prime}\right)$ symmetry inside domains and with the $\mathrm{O}^{\prime}$ (Amam) symmetry at domain walls. A frustration of oxygen octahedral tilts among four domains suggests an un-distorted structure (open circles) for the $Z_{4}$ vortex core. The Glazer notations label the dominant tilting modes at the corresponding locations. (c) A schematic of the domain wall between two antiphase domains, e.g., DW03 between domain $\mathbf{0}$ and $\mathbf{3}$. The open circles indicate the ideal position of oxygens of the T state.

tetragonal state of high- $T_{c}$ superconducting $\mathrm{La}_{2-x} \mathrm{Ba}_{x} \mathrm{CuO}_{4}$, in which the $\mathrm{CuO}_{6}$ octahedra also tilt along the $\left.<100\right\rangle_{T}$ directions $^{12,13}$ and are governed by the $X_{3}^{+}$lattice mode. ${ }^{34}$ We also note that the $\mathrm{T}^{\prime}$ structure has been reported in other bi-layered perovskite magnets such as $\mathrm{Sr}_{2}(\mathrm{Ho}, \mathrm{Y}) \mathrm{Mn}_{2} \mathrm{O}_{7}$ (ref. 35) and $\mathrm{Tb}_{2} \mathrm{Sr}(\mathrm{Fe}, \mathrm{Co})_{2} \mathrm{O}_{7}$ (refs 36-38), and magnetism in (layered) perovskites tends to be strongly coupled with structural distortions, so this unique domain topology may not be limited to structural domains and domain walls in $\left(\mathrm{Ca}_{1}, \mathrm{Sr}_{3} \mathrm{Ti}_{2} \mathrm{O}_{7}\right.$. Our work should initiate further exploration of new types of topological defects.

\section{MATERIALS AND METHODS}

Eleven high-quality polycrystalline specimens of $\mathrm{Ca}_{3-x} \mathrm{Sr}_{x} \mathrm{Ti}_{2} \mathrm{O}_{7}(x=0,0.3,0.5$, $0.6,0.8,0.85,0.9,1,1.5,2$ and 3 ) were prepared using a solid-state reaction method. Stoichiometric mixtures of $\mathrm{CaCO}_{3}$ (Alfa Aesar 99.95\%), $\mathrm{SrCO}_{3}$ (Alfa Aesar 99.99\%) and $\mathrm{TiO}_{2}$ (Alfa Aesar Puratronic 99.995\%) powders were ground, pelletised and then sintered at $1,550-1,660^{\circ} \mathrm{C}$ for $30 \mathrm{~h}$. In the range of $1.1 \leqslant x<1.5$, we found a triple-layered $\mathrm{A}_{4} \mathrm{~B}_{3} \mathrm{O}_{10}$ phase is more stable and favoured than the bi-layered $\mathrm{A}_{3} \mathrm{~B}_{2} \mathrm{O}_{7}$ phase. The powder specimens for acquiring synchrotron X-ray powder diffraction data were sealed in $0.2-\mathrm{mm}$ diameter capillary quartz tubes. All synchrotron X-ray experiments were performed on beamline BL01C2 at the National Synchrotron Radiation Research Center, Taiwan. The powder diffraction patterns were acquired using a collimated synchrotron-radiation beam with the wavelength of $0.688 \AA$ (18 KeV). Powdered sample were loaded into a 0.2-mm capillary for uniform absorption and faster rotation during data collection. The general structure analysis system program using the Rietveld method with a pseudo-Voigt profile function was exploited to analyse the X-ray powder diffraction data. Specimens for transmission electron microscope (TEM) studies were prepared by mechanical polishing, followed by Ar-ion milling, and studied using a JEOL-2010F TEM at Rutgers University, NJ. We observed $Z_{4}$ vortex domains with superlattice DF-TEM imaging taking (1) $S_{1}=1 / 2(130)_{T}=(120)_{\text {orth }}$, (2) $S_{2}=1 / 2(020)_{T}=(110)_{\text {orth }}$ and (3) $S_{3}=1 / 2(130)_{T}=(210)_{\text {orth }}$ spots. In situ heating TEM experiment was carried out using a JEOL-2000FX TEM with a high-temperature specimen holder at National Taiwan University in Taiwan. High-angle annular dark-field scanning TEM imaging with atomic-column resolution was carried out using a JEOL-2100F microscope equipped with a spherical aberration Cs-corrector at National Taiwan University in Taiwan. High-angle annular dark-field images were acquired in the condition: $512 \times 512$ with $0.024 \mathrm{~nm}$ per pixel with collection angle between 80 and 210 mrad. For dielectric constant measurements, two electrodes were prepared using Au sputtering on polished specimens with a capacitor geometry, and an LCR metre at $f=44 \mathrm{kHz}$ was utilised.

Data availability

The authors declare that all source data supporting the findings of this study are available within the article and the file.

\section{ACKNOWLEDGEMENTS}

The work at Rutgers was funded by the Gordon and Betty Moore Foundation's EPiQS Initiative through Grant GBMF4413 to the Rutgers Center for Emergent Materials, and the work at Postech was supported by the Max Planck POSTECH/KOREA Research Initiative Program (grant no. 2011-0031558) through NRF of Korea funded by MSIP.

\section{CONTRIBUTIONS}

F.-T.H. and M.-W.C. conducted the (S)TEM experiments. B.G. and X.L. synthesised single crystals. J.W.K. and Y.W. performed the dielectric measurments. C.-K.C and H.-S. S. acquired the synchroton X-ray data. F.-T.H. and S.-W.C. wrote the paper. S.-W.C. initiated and supervised the research.

\section{COMPETING INTERESTS}

The authors declare no conflict of interest.

\section{REFERENCES}

1. Bednorz, J. G. \& Müller, K. A. Perovskite-type oxides-the new approach to highTc superconductivity. Rev. Mod. Phys. 60, 585-600 (1988).

2. Cheong, S.-W. \& Mostovoy, M. Multiferroics: a magnetic twist for ferroelectricity. Nat. Mater. 6, 13-20 (2007). 
3. Von Hippel, A. Ferroelectricity, domain structure, and phase transitions of barium titanate. Rev. Mod. Phys. 22, 221-246 (1950).

4. Salamon, M. B. \& Jaime, M. The physics of manganites: Structure and transport. Rev. Mod. Phys. 73, 583-628 (2001).

5. Haghiri-Gosnet, A. M. \& Renard, J. P. CMR manganites: physics, thin films and devices. J. Phys. D Appl. Phys. 36, R127-R150 (2003).

6. Uehara, M., Mori, S., Chen, C. H. \& Cheong, S. W. Percolative phase separation underlies colossal magnetoresistance in mixed-valent manganites. Nature 399, 560-563 (1999).

7. Choi, T., Lee, S., Choi, Y. J., Kiryukhin, V. \& Cheong, S. W. Switchable ferroelectric diode and photovoltaic effect in $\mathrm{BiFeO}_{3}$. Science 324, 63-66 (2009).

8. Yang, W. S., Noh, J. H., Jeon, N. J., Kim, Y. C. \& Ryu, S. High-performance photovoltaic perovskite layers fabricated through intramolecular exchange. Science 348, 1234-1237 (2015).

9. Kim, H. J. et al. High mobility in a stable transparent perovskite oxide. Appl. Phys. Express 5, 061102 (2012).

10. Filip, M. R., Eperon, G. E., Snaith, H. J. \& Giustino, F. Steric engineering of metalhalide perovskites with tunable optical band gaps. Nat. Commun. 5, 5757 (2014).

11. Navrotsky, A. Energetics and crystal chemical systematics among ilmenite, lithium niobate, and perovskite structures. Chem. Mater. 10, 2787-2793 (1998).

12. Zhu, Y., Moodenbaugh, A. R., Cai, Z. X., Tafto, J. \& Suenaga, M. Tetragonalorthorhombic structural modulation at low temperature in $\mathrm{La}_{2-\mathrm{x}} \mathrm{Ba}_{\mathrm{x}} \mathrm{CuO}_{4}$. Phys. Rev. Lett. 73, 3026-3029 (1994).

13. Chen, C. H., Cheong, S.-W., Werder, D. J. \& Takagi, H. Microstructural changes induced by lattice instabilities in $\mathrm{La}_{2-\mathrm{x}} \mathrm{Ba}_{\mathrm{x}} \mathrm{CuO}_{4}(\mathrm{x}=0.125)$ and $\mathrm{Nd}_{2} \mathrm{NiO}_{4}$. Physica $\mathrm{C}$ 206, 183-194 (1993)

14. Sergienko, I. A. \& Dagotto, E. Role of the Dzyaloshinskii-Moriya interaction in multiferroic perovskites. Phys. Rev. B 73, 094434 (2006).

15. Reaney, I. M. \& Woodward, D. I. Displacive phase transitions and intermediate structures in perovskites. J. Am. Ceram. Soc. 94, 2242-2247 (2011).

16. Ahart, M. et al. Origin of morphotropic phase boundaries in ferroelectrics. Nature 451, 545-548 (2008).

17. Millis, A. J. Lattice effects in magnetoresistive manganese perovskites. Nature 392, 147-150 (1998).

18. Imada, M., Fujimori, A. \& Tokura, Y. Metal-insulator transitions. Rev. Mod. Phys. 70, 1039-1264 (1998).

19. Mulder, A. T., Benedek, N. A., Rondinelli, J. M. \& Fennie, C. J. Turning $\mathrm{ABO}_{3}$ antiferroelectrics into ferroelectrics: design rules for practical rotation-driven ferroelectricity in double perovskites and $\mathrm{A}_{3} \mathrm{~B}_{2} \mathrm{O}_{7}$ Ruddlesden-Popper compounds. Adv. Funct. Mater. 23, 4810-4820 (2013).

20. Oh, Y. S., Luo, X., Huang, F.-T., Wang, Y. \& Cheong, S.-W. Experimental demonstration of hybrid improper ferroelectricity and the presence of abundant charged walls in $\left(\mathrm{Ca}, \mathrm{Sr}_{3} \mathrm{Ti}_{2} \mathrm{O}_{7}\right.$ crystals. Nat. Mater. 14, 407-413 (2015).

21. Benedek, N. A. \& Fennie, C. J. Hybrid improper ferroelectricity: a mechanism for controllable polarization-magnetization coupling. Phys. Rev. Lett. 106, 107204 (2011).

22. Huang, F.-T. et al. Domain topology and domain switching kinetics in a hybrid improper ferroelectric. Nat. Commun. 7, 11602 (2016).

23. Harris, A. B. Symmetry analysis for the Ruddlesden-Popper systems $\mathrm{Ca}_{3} \mathrm{Mn}_{2} \mathrm{O}_{7}$ and $\mathrm{Ca}_{3} \mathrm{Ti}_{2} \mathrm{O}_{7}$. Phys. Rev. B 84, 064116 (2011).
24. Glazer, A. M. The classification of tilted octahedra in perovskites. Acta Cryst. B 28, 3384-3392 (1972).

25. Senn, M. S. et al. Symmetry switching of negative thermal expansion by chemical control. J. Am. Chem. Soc. 138, 5479-5482 (2016).

26. Senn, M. S. et al. Negative thermal expansion in hybrid improper ferroelectric Ruddlesden-Popper perovskites by symmetry trapping. Phys. Rev. Lett. 114, 035701 (2015)

27. Pitcher, M. J., Mandal, P., Dyer, M. S., Alaria, J. \& Borisov, P. Tilt engineering of spontaneous polarization and magnetization above $300 \mathrm{~K}$ in a bulk layered perovskite. Science 347, 420-424 (2015).

28. Chae, S. C. et al. Self-organization, condensation, and annihilation of topological vortices and antivortices in a multiferroic. Proc. Natl Acad. Sci. USA 107, 21366-21370 (2010)

29. Stokes, H. T. \& Hatch, D. M.. Isotropy Subgroup of the 230 Crystallographic Space Groups. World Scientific: Singapore, (1988).

30. Jia, C.-L. et al. Atomic-scale study of electric dipoles near charged and uncharged domain walls in ferroelectric films. Nat. Mater. 7, 57-61 (2008).

31. Huang, F.-T. et al. Duality of topological defects in hexagonal manganites. Phys. Rev. Lett. 113, 267602 (2014).

32. Choi, T. et al. Insulating interlocked ferroelectric and structural antiphase domain walls in multiferroic $\mathrm{YMnO}_{3}$. Nat. Mater. 9, 253-258 (2010).

33. Yu, X. Z. et al. Real-space observation of a two-dimensional skyrmion crystal. Nature 465, 901-904 (2010).

34. Balachandran, P. V., Puggioni, D. \& Rondinelli, J. M. Crystal-chemistry guidelines for noncentrosymmetric $\mathrm{A}_{2} \mathrm{BO}_{4}$ Ruddlesden-Popper Oxides. Inorg. Chem. 53, 336-348 (2014)

35. Battle, P. D., Millburn, J. E. \& Rosseinsky, M. J. Neutron diffraction study of the structural and electronic properties of $\mathrm{Sr}_{2} \mathrm{HoMn}_{2} \mathrm{O}_{7}$ and $\mathrm{Sr}_{2} \mathrm{YMn}_{2} \mathrm{O}_{7}$. Chem. Mater. 9, 3136-3143 (1997)

36. Sánchez-Andújar, M. \& Señarís-Rodríguez, M. A. Cation ordering and electrical properties of the Ruddlesden-Popper $\mathrm{Gd}_{2-2 x} \mathrm{Sr}_{1+2 x} \mathrm{CO}_{2} \mathrm{O}_{7}$ compounds $(\mathrm{x}=0$ and 0.10). Z. anorg. allg. Chem. 633, 1890-1896 (2007).

37. Hickey, P. J., Knee, C. S., Henry, P. F. \& Weller, M. T. Spin-crossover structural transition in the layered perovskite $\mathrm{Gd}_{2} \mathrm{SrCO}_{2} \mathrm{O}_{7}$. Phys. Rev. B 75, 024113 (2007).

38. Samaras, D., Collomb, A. \& Joubert, J. C. Determination des structures de deux ferrites mixtes nouveaux de formule $\mathrm{BaLa}_{2} \mathrm{Fe}_{2} \mathrm{O}_{7}$ et $\mathrm{SrTb}_{2} \mathrm{Fe}_{2} \mathrm{O}_{7}$. J. Solid State Chem. 7, 337-348 (1973).

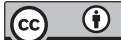

This work is licensed under a Creative Commons Attribution 4.0 International License. The images or other third party material in this article are included in the article's Creative Commons license, unless indicated otherwise in the credit line; if the material is not included under the Creative Commons license, users will need to obtain permission from the license holder to reproduce the material. To view a copy of this license, visit http://creativecommons.org/licenses/ by/4.0/

(c) The Author(s) 2016

Supplementary Information accompanies the paper on the npj Quantum Materials website (http://www.nature.com/npjquantmats) 